\title{
New and confirmed records of fruit flies (Diptera, Tephritidae) from Italy
}

\author{
Luca Mazzon‡, Daniel Whitmore§, Pierfilippo Cerrettil, Valery A. Korneyev『 \\ $\ddagger$ Department of Agronomy, Food, Natural Resources, Animals and Environment (DAFNAE), University of Padua, Padua, Italy \\ $\S$ Staatliches Museum für Naturkunde Stuttgart, Stuttgart, Germany \\ | Department of Biology and Biotechnology 'Charles Darwin', Sapienza University of Rome, Rome, Italy \\ II I.I.Schmalhausen Institute of Zoology, Kyiv, Ukraine
}

Corresponding author: Luca Mazzon (Imazzon@unipd.it)

Academic editor: AJ Fleming

Received: 28 May 2021 | Accepted: 21 Jul 2021 | Published: 31 Aug 2021

Citation: Mazzon L, Whitmore D, Cerretti P, Korneyev VA (2021) New and confirmed records of fruit flies

(Diptera, Tephritidae) from Italy. Biodiversity Data Journal 9: e69351. https://doi.org/10.3897/BDJ.9.e69351

\section{Abstract}

\section{Background}

Prior to this study, 141 species of Tephritidae were known to occur in Italy.

\section{New information}

Italian records of nine species of the family Tephritidae (Diptera) are provided. Five species, Eurasimona stigma (Loew, 1840), Noeeta bisetosa Merz, 1992, Campiglossa doronici (Loew, 1856), Xyphosia laticauda (Meigen, 1826) and Rhagoletis berberidis Jermy, 1961 are recorded from Italy for the first time, whereas four species, Inuromaesa maura (Frauenfeld, 1857), Urophora cuspidata (Meigen, 1826), Tephritis conyzifoliae Merz, 1992 and T. mutabilis Merz, 1992, previously recorded in the Fauna Europaea database without reference to collection material, are confirmed and supplemented with host plant data and other collection data. 


\section{Keywords}

Diptera, Tephritidae, Italy, checklist, additions

\section{Introduction}

Tephritidae is one of the largest families of cyclorrhaphous Diptera, with almost 5,000 named species predominantly found in the tropics (A. Norrbom, pers. comm.). The family is also referred to as "fruit flies", as it contains many species that are specialised feeders on fleshy fruit. The tropical regions are true hotspots of tephritid diversity, including hundreds of fruit-eating species, but also species with saprophagous larvae feeding under the bark of fallen trees or in bamboo culms (Korneyev 1999, Dohm et al. 2014). On the other hand, most Palaearctic species are flower and seed feeders, with larvae that are borers in the flower heads, stems and rhizomes of Asteraceae, Lamiaceae and Acanthaceae. The European fauna is represented by at least 265 species belonging to three subfamilies: Dacinae, Tephritinae and Trypetinae (Merz and Korneyev 2011, V. Korneyev, unpublished data). Some of the fruit-eating species are widespread economic pests which cause serious damage to fruit crops and stored fruit. The olive fly, Bactrocera oleae (Rossi, 1794) and the medfly, Ceratitis capitata (Wiedemann, 1817), as well as many members of the genera Rhagoletis Loew, 1862 and Carpomya Costa, 1854, are well-known examples.

Starting from Rondani (Rondani 1856, Rondani 1870), who listed 109 nominal species known from Italy and following numerous nomenclatural changes, the number of Italian fruit fly species reached 133 species according to Belcari et al. (1995). Later, Merz and Korneyev (2011) increased this number to 141 species, but did not provide occurrence data for species added to the Italian list. These were: Inuromaesa maura (Frauenfeld, 1857), Urophora congrua Loew, 1862, U. cuspidata (Meigen, 1826), Tephritis conyzifoliae Merz, 1992, T. maccus Hering, 1937, T. mutabilis Merz, 1992, Chaetorellia acrolophi White \& Marquardt, 1989, Terellia (Cerajocera) rhapontici Merz, 1990, Rhagoletis batava Hering, 1958 and $R$. cingulata (Loew, 1862), all based on collection material identified by Bernhard Merz, but not formally published.

Other relatively recent contributions to the Italian tephritid fauna, including first Italian records, were made by Merz (2002) for Campiglossa misella (Loew, 1869) and Rivosecchi (2008) for Euphranta toxoneura (Loew, 1846). Gentilini et al. (2006) described two fossil species from the Upper Miocene (Messinian) of Monte Castellaro. Seljak (2013) recorded Euaresta aequalis (Loew), a non-native, North American species considered beneficial as a biological control agent of the invasive plant Xanthium strumarium L. (Asteraceae), from Italy and Slovenia for the first time. Recently, Nugnes et al. (2018) provided the first Italian records of the invasive fruit pest species Bactrocera dorsalis (Hendel, 1912) from the Region of Campania.

In this paper, we record five tephritid species for Italy for the first time and confirm the occurrence in the country of four additional species, based on detailed collection data and host plant information obtained during research on symbiotic bacteria of over 30 fruit fly 
species. The analysis revealed the presence of hereditary symbiotic bacteria in Bactrocera oleae (Rossi, 1790) (Capuzzo et al. 2005) and in all studied species of the tribe Tephritini and the genus Noeeta Robineau-Desvoidy, 1830. In other tribes (e.g., Myopitini, Xyphosiini and Terelliini), despite the common trait of larvae developing in Asteraceae flower heads, evolution does not seem to have occurred for the establishment of a hereditary bacterial interaction (Mazzon et al. 2008, Mazzon et al. 2010, Mazzon et al. 2011).

\section{Materials and methods}

The flies were collected in Italy by the first author, reared from mature larvae and pupae collected together with infested flower heads. Flower heads were detached from their stems and placed in net bags at room temperature to allow the insects to complete their development. An in-field pre-screening of positive samples involved sectioning of the inflorescences and inspection for the presence of larvae or pupae. Adults of Eurasimona stigma (Loew, 1840) were collected with a mouth aspirator while resting on their host plant.

The species were identified using the keys of White (1988) and Merz (1994). The nomenclature follows Norrbom et al. (1999). Host plants were determined using the Italian botanical keys in Pignatti (1982); when necessary, identifications were confirmed by a specialist.

All voucher specimens (Figs 1,2) are deposited in the fruit fly collection of the Laboratory of Entomology of the Department of Agronomy, Food, Natural Resources, Animals and Environment, University of Padua, Italy (UPI).

\section{Taxon treatments}

\section{Eurasimona stigma (Loew, 1840)}

\section{Material}

a. $\quad$ scientificName: Eurasimona stigma (Loew, 1840); higherClassification: Subfamily Tephritinae, Tribe Myopitini; genus: Eurasimona; specificEpithet: stigma; scientificNameAuthorship: (Loew, 1840); continent: Europe; country: Italy; countryCode: I; stateProvince: Veneto Region; county: Padova Province; locality: Euganean Hills;

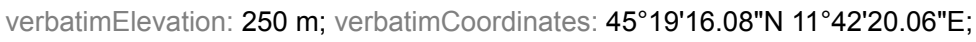
decimalLatitude: 45.3211; decimalLongitude: 11.7055; georeferenceSources: Google Maps; samplingProtocol: mouth aspirator; eventDate: 14/06/2006; habitat: edge of forest, on Achillea flowers; individualCount: 3; sex: male; lifeStage: adult; preparations: dry; recordedBy: L. Mazzon; identifiedBy: L. Mazzon; dateldentified: 2006; basisOfRecord: PreservedSpecimen

\section{Distribution}

Austria, Czechia, Estonia, Germany, Finland, France, Hungary, Latvia, Lithuania, North Macedonia, Moldova, Slovakia, Sweden, Ukraine (Korneyev and White 1991, Merz and 
Korneyev 2011); Russia, east to southern Siberia (Krasnoyarsk); Kazakhstan; Turkmenistan; Uzbekistan; Kyrghyzstan (Korneyev and White 1991); Iran (Zarghani et al. 2016). The species (Fig. 1a) is here recorded as new to Italy.

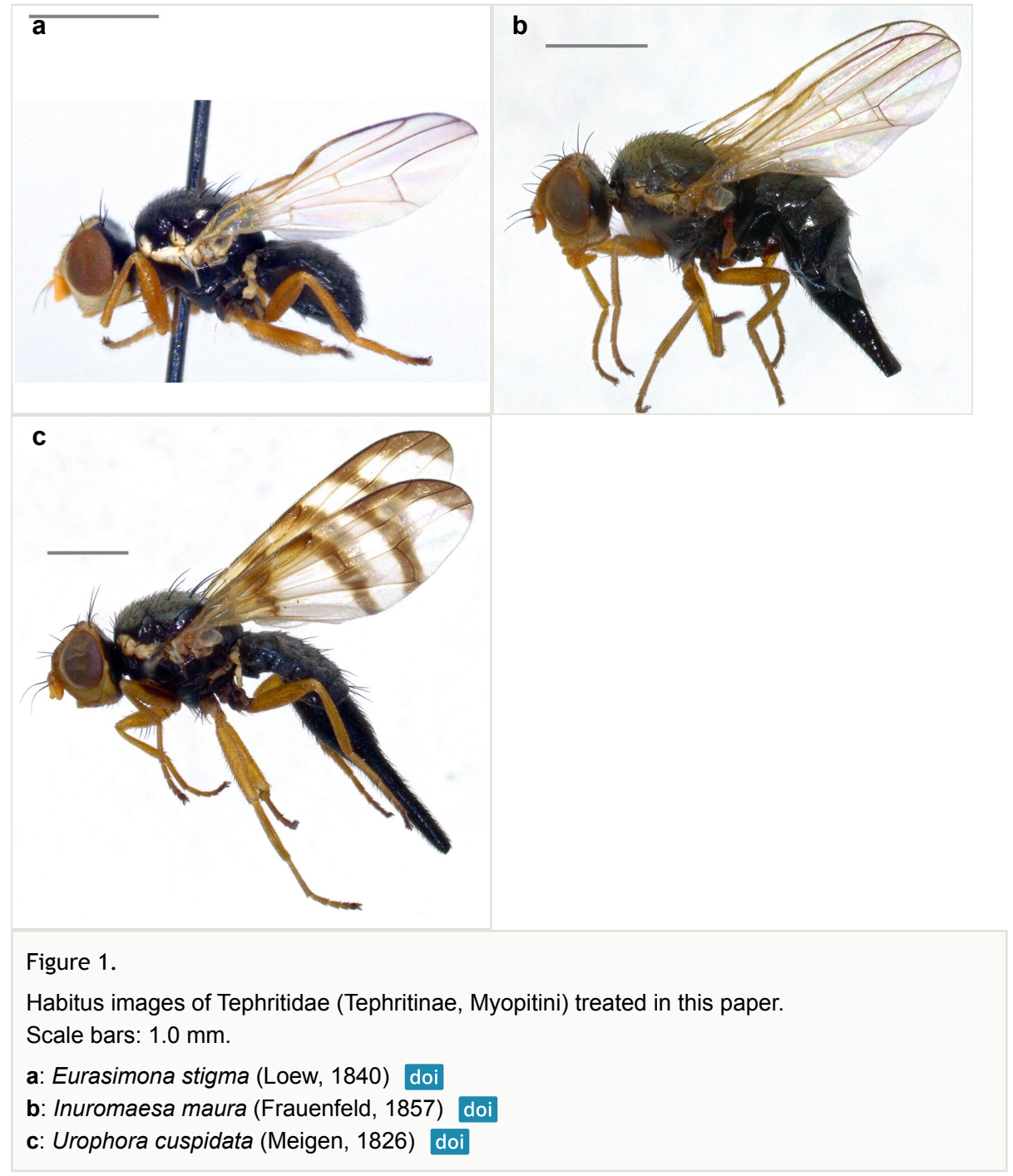

\section{Biology}

The biology of E. stigma is poorly known. The flies are said to have been reared from Leucanthemum vulgare Lam. (Roser 1840, as "Chrysanthemum leucanthemum"), Achillea millefolium L. (Loew 1862: 68), Anthemis arvensis L. (Korneyev et al. 2005; forming small non-lignified galls in a single flower head), A. cotula L. (Hendel 1927) and Tanacetum vulgare L. (Merz 1994), but there are no comprehensive rearing data or descriptions of its biology. 


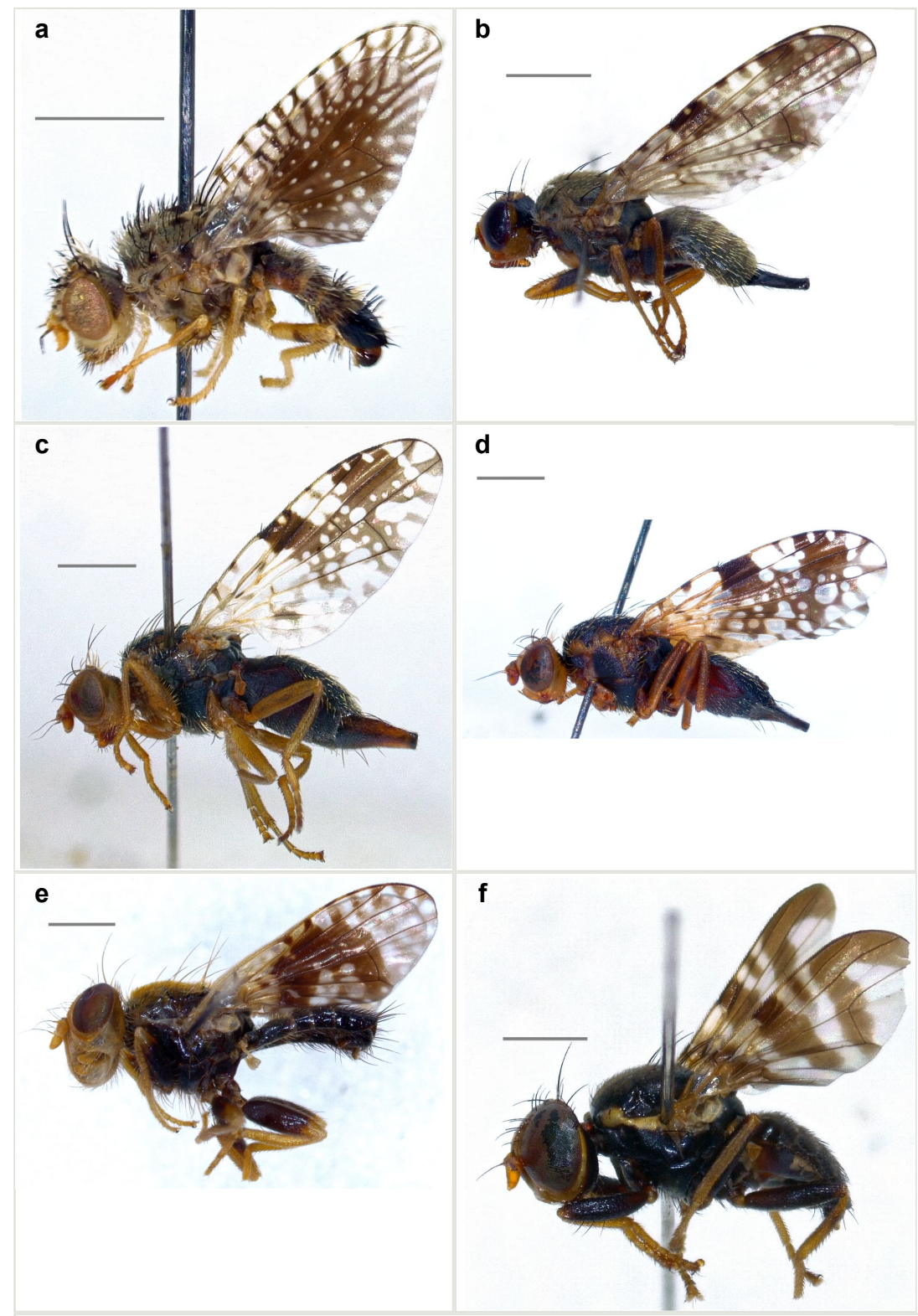

Figure 2.

Habitus images of Tephritidae treated in this paper. a. Tephritinae, Noeetini; b-d Tephritinae, Tephritini; e. Tephritinae, Xyphosiini; f. Trypetinae, Carpomyini. Scale bars: $1.0 \mathrm{~mm}$.
a: Noeeta bisetosa Merz, 1992 doi
b: Campiglossa doronici (Loew, 1856) doi
c: Tephritis conyzifoliae Merz, 1992 doi
d: Tephritis mutabilis Merz, 1992 doi
e: Xyphosia laticauda (Meigen, 1826) doi
f: Rhagoletis berberidis Jermy, 1961 doi 


\section{Inuromaesa maura (Frauenfeld, 1857)}

\section{Material}

a. ScientificName: Inuromaesa maura (Frauenfeld, 1857); higherClassification: Subfamily Tephritinae, Tribe Myopitini; genus: Inuromaesa; specificEpithet: maura; scientificNameAuthorship: (Frauenfeld, 1857); continent: Europe; country: Italy; countryCode: I; stateProvince: Friuli-Venezia Giulia Region; county: Pordenone Province; locality: Montereale Val Cellina; verbatimElevation: $1100 \mathrm{~m}$; verbatimCoordinates: $46^{\circ} 10^{\prime} 00.0^{\prime \prime} \mathrm{N} 12^{\circ} 36^{\prime} 00.0 " \mathrm{E}$; decimalLatitude: 46.1668; decimalLongitude: 12.6000; georeferenceSources: Google Maps; samplingProtocol: from flower heads of Inula hirta; eventDate: 25/06/2005; habitat: edge of forest; individualCount: 5; sex: 4 males and 1 female; lifeStage: adult, reared from immature stages; preparations: dry whole insect; recordedBy: L. Mazzon; identifiedBy: L. Mazzon; dateldentified: 2005; basisOfRecord: PreservedSpecimen

\section{Distribution}

Austria, Czechia, central and southern France, Hungary, Italy, Slovakia, northern Spain, Switzerland and Ukraine (Merz and Korneyev 2011); Russia: West Siberia; Kazakhstan (Korneyev and White 1999); Iran (Zarghani et al. 2016). Note: the present records from Italy (Fig. 1b) confirm the country-level record by Merz and Korneyev (2011).

\section{Biology}

The larvae develop in the achenes of Pentanema hirtum (L.) D.Gut.Larr. et al. (= Inula hirta), Pentanema oculus-christi (L.) D.Gut.Larr. et al. (= Inula oculus-christi) (Frauenfeld 1857), Pentanema montanum (L.) D.Gut.Larr. et al. (= Inula montana) (Anonymous 1934), Pentanema ensifolium (L.) D.Gut.Larr. et al. (= Inula ensifolia) (Mihalyi 1960, Korneyev and White 1991) and Pentanema salicinum (L.) D.Gut.Larr. et al. (= Inula salicina) (Richter 1988), forming no obvious galls.

\section{Urophora cuspidata (Meigen, 1826)}

\section{Materials}

a. ScientificName: Urophora cuspidata (Meigen, 1826); higherClassification: Subfamily Tephritinae, Tribe Myopitini; genus: Urophora; specificEpithet: cuspidata;

scientificNameAuthorship: (Meigen, 1826); continent: Europe; country: Italy; countryCode: I; stateProvince: Friuli-Venezia Giulia Region; county: Pordenone Province; municipality: Fanna; locality: Via Vals; verbatimElevation: $320 \mathrm{~m}$; verbatimCoordinates: 46¹1'6.43" $\mathrm{N}$ 1243'55.33"E; decimalLatitude: 46.1851; decimalLongitude: 12.7320; georeferenceSources: Google Maps; samplingProtocol: reared from flower heads of Centaurea scabiosa; eventDate: 15/07/2006; habitat: grassland; individualCount: 2; sex: 1 male and 1 female; lifeStage: adult; preparations: dry whole insect; recordedBy: V. Girolami; dateldentified: 2007; basisOfRecord: PreservedSpecimen

b. $\quad$ scientificName: Urophora cuspidata (Meigen, 1826); higherClassification: Subfamily Tephritinae, Tribe Myopitini; genus: Urophora; specificEpithet: cuspidata; 
scientificNameAuthorship: (Meigen, 1826); continent: Europe; country: Italy; countryCode: I; stateProvince: Friuli-Venezia Giulia Region; county: Pordenone Province; municipality: Fanna; locality: Via Vals; verbatimElevation: $320 \mathrm{~m}$; verbatimCoordinates: 46¹1'6.43" $\mathrm{N}$ 12²'55.33"E; decimalLatitude: 46.1851; decimalLongitude: 12.7320;

georeferenceSources: Google Maps; samplingProtocol: reared from flower heads of Centaurea scabiosa; eventDate: 26/06/2007; habitat: grassland; individualCount: 2; sex: 2 females; preparations: dry whole insect; recordedBy: V. Girolami; dateldentified: 2007; basisOfRecord: PreservedSpecimen

\section{Distribution}

Northern, central and eastern Europe from northern Spain, southern France and Italy (Merz 1994, Merz and Korneyev 2011) to Ukraine, European and Asian Russia and Kazakhstan (Korneyev and White 1996); Iran (Mohamadzade Namin and Nozari 2011). Notes: the present records from Italy (Fig. 1c) confirm the country-level record by Merz and Korneyev (2011). The record from Turkey by Kütük et al. (2013) needs confirmation.

\section{Biology}

The larvae develop in flower heads of Centaurea scabiosa L. (incl. ssp. alpestris) and Ce. collina L. (White and Korneyev 1989); records of other host species, including "Ce. tenuifolia" (Merz 1994) need confirmation.

\section{Noeeta bisetosa Merz, 1992}

\section{Material}

a. ScientificName: Noeeta bisetosa Merz, 1992; higherClassification: Subfamily Tephritinae, Tribe Noeetini; genus: Noeeta; specificEpithet: bisetosa; scientificNameAuthorship: Merz, 1992; continent: Europe; country: Italy; countryCode: I; stateProvince: Friuli-Venezia Giulia Region; county: Pordenone Province; municipality: Fanna; locality: Val Cellina; verbatimElevation: $290 \mathrm{~m}$; verbatimCoordinates: $46^{\circ} 10^{\prime} 11.8^{\prime \prime} \mathrm{N} 12^{\circ} 40^{\prime} 02.7^{\prime \prime E}$; decimalLatitude: 46.1699; decimalLongitude: 12.6674; georeferenceSources: Google Maps; samplingProtocol: reared from flower heads of Hieracium piloselloides; eventDate: 10/07/2006; habitat: gravel streambed; individualCount: 16; sex: 9 males and 7 females; lifeStage: adult; preparations: dry whole insect; recordedBy: V. Girolami; identifiedBy: L. Mazzon; dateldentified: 2006; basisOfRecord: PreservedSpecimen

\section{Distribution}

Austria (Merz and Kofler 2008), Hungary (Merz 2000), Russia (Basov 1999), Switzerland (Merz 1992) and Ukraine (Korneyev 2003). The species (Fig. 2a) is here recorded as new to Italy.

\section{Biology}

The larvae feed in flower heads of Hieracium piloselloides Vill. (Merz 1992). 


\section{Campiglossa doronici (Loew, 1856)}

\section{Material}

a. $\quad$ scientificName: Campiglossa doronici (Loew, 1856); higherClassification: Subfamily Tephritinae, Tribe Tephritini; genus: Campiglossa; specificEpithet: doronici; scientificNameAuthorship: (Loew, 1856); continent: Europe; country: Italy; countryCode: I; stateProvince: Veneto Region; county: Vicenza Province; locality: Monte Cengio;

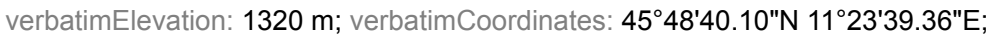
decimalLatitude: 45.8111; decimalLongitude: 11.3943; georeferenceSources: Google Maps; samplingProtocol: reared from flower heads of Doronicum austriacum; eventDate: 02/07/2005; habitat: edge of forest; individualCount: 20; sex: 11 males and 9 females; lifeStage: adult; preparations: dry whole insect; recordedBy: L. Mazzon; identifiedBy: L. Mazzon; dateldentified: 2005; basisOfRecord: PreservedSpecimen

\section{Distribution}

Austria, Czechia, France, Poland, Romania, Slovakia and Ukraine (Merz and Korneyev 2011). The species (Fig. 2b) is here recorded as new to Italy.

\section{Biology}

The larvae feed in flower heads of Doronicum austriacum Jacq. (Loew 1856).

\section{Tephritis conyzifoliae Merz, 1992}

\section{Material}

a. $\quad$ scientificName: Tephritis conyzifoliae Merz, 1992; higherClassification: Subfamily Tephritinae, Tribe Tephritini; genus: Tephritis; specificEpithet: conyzifoliae; scientificNameAuthorship: Merz, 1992; continent: Europe; country: Italy; countryCode: I; stateProvince: Trentino-Alto Adige Region; county: Trento Province; municipality: Moena; locality: San Pellegrino Pass; verbatimElevation: $1925 \mathrm{~m}$; verbatimCoordinates:

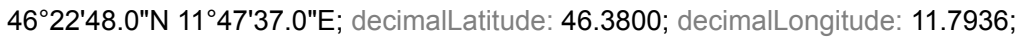
georeferenceSources: Google Maps; samplingProtocol: reared from flower heads of Crepis conyzifolia; eventDate: 28/07/2008; habitat: pasture; individualCount: 17 ; sex: 8 males and 9 females; lifeStage: adult; preparations: dry whole insect; recordedBy: L. Mazzon; identifiedBy: L. Mazzon; dateldentified: 2008; basisOfRecord: PreservedSpecimen

\section{Distribution}

Armenia (Evstigneev and Glukhova 2020), Czechia, France, Italy, Switzerland (Merz and Korneyev 2011), Russia (Evstigneev 2016), Kazakhstan, Kyrgyzstan (Korneyev 2016a), Poland (Klasa and Palaczyk 2005), Tajikistan (Korneyev and Korneyev 2019) and Ukraine (Korneyev and Klasa 2016). Note: the present records from Italy (Fig. 2c) confirm the country-level record by Merz and Korneyev (2011). 


\section{Biology}

The larvae develop in flower heads of Crepis conyzifolia (Gouan) A. Kern. (Merz 1992), Cr. sibirica L. (Shcherbakov 2001, Korneyev 2016a), Cr. pannonica (Jacq.) K. Koch (Evstigneev 2016) and Cr. ciliata K. Koch (Evstigneev and Glukhova 2020).

\section{Notes}

This species was recorded from continental Italy by Merz and Korneyev (2011), without further collection data. Korneyev (2016a) treated T. conyzifoliae as a senior synonym of Tephritis academica Bassov and Tolstoguzova, 1994, T. nartshukovi Bassov and Tolstoguzova, 1994 and T. epicrepis Scherbakov, 2001, all described from Russia.

\section{Tephritis mutabilis Merz, 1992}

\section{Material}

a. $\quad$ scientificName: Tephritis mutabilis Merz, 1992; higherClassification: Subfamily Tephritinae, Tribe Tephritini; genus: Tephritis; specificEpithet: mutabilis; scientificNameAuthorship: Merz, 1992; continent: Europe; country: Italy; countryCode: I; stateProvince: Friuli-Venezia Giulia Region; county: Pordenone Province; municipality: Fanna; verbatimElevation: $280 \mathrm{~m}$; verbatimCoordinates: 46¹1'12.3"N 1244'39.7"E; decimalLatitude: 46.1867; decimalLongitude: 12.7444; georeferenceSources: Google Maps; samplingProtocol: reared from flower heads of Leontodon hispidus; eventDate: 28/05/2007; habitat: grassland; individualCount: 3; sex: 1 males and 2 females; lifeStage: adult; preparations: dry whole insect; recordedBy: V. Girolami; identifiedBy: L. Mazzon; dateldentified: 2007; basisOfRecord: PreservedSpecimen

\section{Distribution}

Central Europe (Austria, Czechia, France, Germany, Italy, Poland, Slovakia, Switzerland) (Merz and Korneyev 2011), Finland (Kahanpää and Winqvist 2014), Russia (Korneyev 2016b) and Ukraine (Korneyev and Klasa 2016). Note: the present records from Italy (Fig. 2d) confirm the country-level record by Merz and Korneyev 2011.

\section{Biology}

The larvae feed in flower heads of Leontodon hispidus L. (Merz 1992, Merz 1994).

\section{Xyphosia laticauda (Meigen, 1826)}

\section{Materials}

a. ScientificName: Xyphosia laticauda (Meigen, 1826); higherClassification: Subfamily Tephritinae, Tribe Xyphosiini; genus: Xyphosia; specificEpithet: Iaticauda; scientificNameAuthorship: (Meigen, 1826); continent: Europe; country: Italy; countryCode: I; stateProvince: Friuli-Venezia Giulia Region; county: Pordenone Province; municipality: Maniago; locality: Colvera; verbatimElevation: 210 m; verbatimCoordinates: 
$46^{\circ} 10^{\prime} 11.11 " \mathrm{~N}, 12^{\circ} 44^{\prime} 16.48 " \mathrm{E}$; decimalLatitude: 46.1698 ; decimalLongitude: 12.7379 ; georeferenceSources: Google Maps; samplingProtocol: reared from flower heads of Centaurea triumfettii; eventDate: 10/07/2006; habitat: gravel streambed; individualCount: 1; sex: male; lifeStage: adult; preparations: dry whole insect; recordedBy: V. Girolami; identifiedBy: L. Mazzon; dateldentified: 2006; basisOfRecord: PreservedSpecimen b. ScientificName: Xyphosia laticauda (Meigen, 1826); higherClassification: Subfamily Tephritinae, Tribe Xyphosiini; genus: Xyphosia; specificEpithet: laticauda; scientificNameAuthorship: (Meigen, 1826); continent: Europe; country: Italy; countryCode: I; stateProvince: Friuli-Venezia Giulia Region; county: Pordenone Province; municipality: Cavasso Nuovo; locality: Meduna; verbatimElevation: $147 \mathrm{~m}$; verbatimCoordinates:

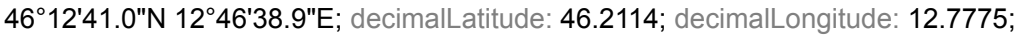
georeferenceSources: Google Maps; samplingProtocol: reared from flower heads of Centaurea triumfettii; eventDate: 07/07/2006; habitat: gravel streambed; individualCount: 1; sex: male; lifeStage: adult; preparations: dry whole insect; recordedBy: V. Girolami; identifiedBy: L. Mazzon; dateldentified: 2006; basisOfRecord: PreservedSpecimen

\section{Distribution}

Austria, France, Hungary, Switzerland (Merz and Korneyev 2011); Armenia (Korneyev 1983); Russian North Caucasus (Korneyev and Korneyev, unpublished data). The species (Fig. 2e) is here recorded as new to Italy.

\section{Biology}

The larvae develop in flower heads of Centaurea montana L. (Frauenfeld 1857); this species is superficially similar to Centaurea triumfettii All.; either both species are infested by this species or misidentifications have occurred.

\section{Rhagoletis berberidis Jermy, 1961}

\section{Material}

a. $\quad$ scientificName: Rhagoletis berberidis Jermy, 1961; higherClassification: Subfamily Trypetinae, Tribe Carpomyini; genus: Rhagoletis; specificEpithet: berberidis; scientificNameAuthorship: Jermy, 1961; continent: Europe; country: Italy; countryCode: I; stateProvince: Friuli-Venezia Giulia Region; county: Pordenone Province; municipality: Claut; verbatimElevation: $675 \mathrm{~m}$; verbatimCoordinates: 46¹6'6.14"N, 12³1'39.23"E; decimalLatitude: 46.2684; decimalLongitude: 12.5276; georeferenceSources: Google Maps; samplingProtocol: reared from fruits of Berberis vulgaris; eventDate: 15/08/2008; habitat: edge of forest; individualCount: 2; sex: males; lifeStage: adult; preparations: dry whole insect; recordedBy: V. Girolami; identifiedBy: L. Mazzon; dateldentified: 2009; basisOfRecord: PreservedSpecimen

\section{Distribution}

Austria, Hungary, Slovakia, Switzerland, Ukraine (Merz and Korneyev 2011); Russian North Caucasus (Kandybina 1977), Turkey (Kütük and Özaslan 2006) and Iran (Mohamadzade Namin et al. 2010). The species (Fig. 2f) is here recorded as new to Italy. 


\section{Biology}

The larvae develop in seeds of Berberis vulgaris L. (Jermy 1961, Merz 1994).

\section{Discussion}

Based on the present results and on a recent revision of the fauna, the revised checklist of Italian Tephritidae (Mazzon and Korneyev, in press) includes 151 extant and two fossil species.

\section{Acknowledgements}

We appreciate the assistance of B. Merz in the identification of some species. We thank V. Girolami for his insights during collection of the specimens and L. Marini (University of Padua-DAFNAE) for confirming some host plant identifications.

\section{Author contributions}

LM: collection and identification of the species, curation of specimens for permanent storage, preparation of the manuscript and photographs; DW: writing, reviewing and editing of manuscript; PC: reviewing and editing of manuscript; VAK: validation of fruit fly identifications, data curation, writing, reviewing and editing of manuscript.

\section{References}

- $\quad$ Basov VM (1999) Tephritidae (Diptera) of Middle Volga and Cis-Ural areas. Vestnik Udmurtskogo Universiteta. Seria Biologicherskoe Raznoobrazie Udmurtskoy Republiki 2: 33-45. [In Russian].

- $\quad$ Belcari A, Girolami V, Rivosecchi L, Zaitzev VF (1995) 72. Diptera Tephritoidea. In: Minelli A, Ruffo S, La Posta S (Eds) Checklist delle specie della fauna italiana. 1-13. Calderini, Bologna.

- $\quad$ Capuzzo C, Firrao G, Mazzon L, Squartini A, Girolami V (2005) 'Candidatus Erwinia dacicola', a coevolved symbiotic bacterium of the olive fly Bactrocera oleae (Gmelin). International Journal of Systematic and Evolutionary Microbiology 55 (4): 1641-1647. https://doi.org/10.1099/ijs.0.63653-0

- Dohm P, Kovac D, Freidberg A, Rull J, Aluja M (2014) Basic biology and host use patterns of tephritid flies (Phytalmiinae: Acanthonevrini, Dacinae: Gastrozonini) breeding in bamboo (Poaceae: Bambusoideae). Annals of the Entomological Society of America 107 (1): 184-203. https://doi.org/10.1603/AN13083

- Evstigneev DA (2016) Tephritid flies of the Higher Tephritines group (Diptera, Tephritidae, Tephritinae) of Ulyanovsk and Samara Regions (Russia). Ukrainska Entomofaunistyka 7 (1): 1-29. [In Russian]. 
- Evstigneev DA, Glukhova NV (2020) First records of two species of Tephritidae and one species of Platystomatidae (Diptera) from Transcaucasia. Zoosystematica Rossica 29 (1): 155-161. https://doi.org/10.31610/zsr/2020.29.1.155

- $\quad$ Frauenfeld GR (1857) Beitrage zur Naturgeschichte der Trypeten nebst 1537 Beschreibung einiger neuer Arten. Sitzungsberichte der Akademie der Wissenschaften in Wien 22: 523-557.

- $\quad$ Gentilini G, Korneyev VA, Kameneva EP (2006) Fossil tephritoid flies (Diptera: Pallopteridae, Ulidiidae, Tephritidae) from the Upper Miocene of Monte Castellaro, Italy, and a review of fossil European tephritoids. Instrumentas Biodiversitatis, Geneva 7: 85-104.

- Hendel F (1927) 49. Trypetidae, Ifg. In: Lindner E (Ed.) Die Fliegen der palaearktischen Region. 5. Stuttgart, 1-221 pp.

- Jermy T (1961) Eine neue Rhagoletis-Art (Diptera: Trypetidae) aus den Fruchten von Berberis vulgaris L. Acta Zoologica Hungarica 7: 133-137.

- Kahanpää J, Winqvist K (2014) Checklist of the Diptera superfamilies Tephritoidea and Sciomyzoidea of Finland (Insecta). ZooKeys 441: 259-275. https://doi.org/10.3897/ zookeys.441.7143

- $\quad$ Kandybina MN (1977) Larvae of fruit-infesting fruit flies (Diptera, Tephritidae). Opredeliteli po Faune SSSR 114: 1-210.

- Klasa A, Palaczyk A (2005) Nasionnicowate (Tephritidae, Diptera) polskich Karpat stan poznania. Biuletyn Muzeum Przyrodniczego w Krakowie 3: 54.

- Korneyev SV (2016a) On the taxonomic revision of the genus Tephritis (Diptera, Tephritidae): new synonymy. Vestnik Zoologii 50 (1): 31-38. https://doi.org/10.1515/ vzoo-2016-0004

- $\quad$ Korneyev SV (2016b) Tephritis mutabilis Merz (Diptera: Tephritidae): first record from Asia. Ukrainska Entomofaunistyka 7 (1): 46.

- Korneyev SV, Klasa A (2016) New records and a revised checklist of fruit flies of the genus Tephritis (Diptera, Tephritidae) from Ukraine. Vestnik Zoologii 50 (3): 219-224. https://doi.org/10.1515/vzoo-2016-0025

- Korneyev SV, Korneyev VA (2019) Revision of the Old-World species of the genus Tephritis (Diptera, Tephritidae) with a pair of isolated apical spots. Zootaxa 4584 (1): 1-73. https://doi.org/10.11646/zootaxa.4584.1.1

- Korneyev VA (1983) Xyphosia laticauda Mg., a species of tephritid flies (Diptera: Tephritidae) new for the USSR fauna. Vestnik zoologii 2: 54.

- Korneyev VA, White IM (1991) Fruit flies of the genus Urophora R.-D. (Diptera, Tephritidae) of the East Palearctic. I. A key to subgenera and review of species (except the subgenus Urophora s. str. Entomological Review (Washington 70 (8): 117-132.

- $\quad K o r n e y e v$ VA, White IM (1996) Fruit-flies of the genus Urophora R.-D. (Diptera: Tephritidae) of Eastern Palaearctics. II. Review of species of the subgenus Urophora s. str. Communication 3. Entomological Review (Washington) 76 (4): 499-513.

- Korneyev VA (1999) Phylogenetic relationships among higher groups of Tephritidae. In: Aluja M, Norrbom AL (Eds) Fruit flies (Tephritidae): Phylogeny and evolution of behavior. CRC, Boca Raton, 73-113 pp. https://doi.org/10.1201/9781420074468.sec2

- Korneyev VA, White IM (1999) Tephritidae of the genus Urophora R.-D. (Diptera, Tephritidae of east palaearctic: III. Key to palaearctic species. Entomological Review 79 (3). 
- Korneyev VA (2003) New and little-known Tephritidae (Diptera, Cyclorrhapha) from Europe. Vestnik Zoologii 37 (3): 3-12.

- Korneyev VA, Zwölfer H, Seitz A (2005) Phylogenetic relationships, ecology and ecological genetics of cecidogeneous Tephritidae. In: Raman A, Schaefer CW, Withers TM (Eds) Biology, ecology and evolution of gall-inducing arthropods. Science Publishers, Enfield, New Hampshire, USA, 321-371 pp.

- Kütük M, Özaslan M (2006) Faunistical and systematical studies on the Trypetinae (Diptera: Tephritidae) in the Turkey along with a new record to Turkish fauna. Munis Entomology and Zoology 1: 173-178.

- Kütük M, Yaran M, Hayat R, Koyuncu M, Görmez V, Aytekin HU (2013) The determination of fruit fly (Diptera: Tephritidae) fauna in Adyaman, Kilis, and anlurfa provinces with a new record for Turkish fauna. Turkish Journal of Zoology 37: 38-49. https://doi.org/10.3906/zoo-1111-9

- $\quad$ Loew H (1856) Neue Beitrage zur Kenntniss der Dipteren. Vierter 3029 Beitrag. Programm K. Realschule Meseritz

- $\quad$ Loew H (1862) Die europäische Bohrfliegen (Trypetidae). Wien, 1-128 + I-XXVI pp.

- Mazzon L, Piscedda A, Simonato M, Martinez-Sañudo I, Squartini A, Girolami V (2008) Presence of specific symbiotic bacteria in flies of the subfamily Tephritinae (Diptera Tephritidae) and their phylogenetic relationships: proposal of Candidatus Stammerula tephritidis. International Journal of Systematic and Evolutionary Microbiology 58 (6). https://doi.org/10.1099/ijs.0.65287-0

- Mazzon L, Martinez-Sañudo I, Simonato M, Squartini A, Savio C, Girolami V (2010) Phylogenetic relationships between flies of the Tephritinae subfamily (Diptera, Tephritidae) and their symbiotic bacteria. Molecular Phylogenetics and Evolution 56 (1): 312-326. https://doi.org/10.1016/j.ympev.2010.02.016

- Mazzon L, Martinez-Sañudo I, Savio C, Simonato M, Squartini A (2011) Stammerula and other symbiotic bacteria within the fruit flies inhabiting Asteraceae flowerheads. In: Zchori-Fein E, Bourtzis K (Eds) Manipulative tenants: bacteria associated with arthropods,. CRC Press Taylor \& Francis [ISBN 9781138374331].

- Merz B (1992) Fünf neue Fruchtfliegenarten aus den Schweizer Alpen und systematische Bemerkungen zu einigen europischen Gattungen und Arten (Diptera, Tephritidae). Mitteilungen der Schweizerischen Entomologischen Gesellschaft 65: 227-239.

- Merz B (1994) Diptera: Tephritidae. Insecta Helvetica Fauna. 10. HGE Press, Geneva, 198 pp.

- Merz B (2000) Additions and corrections to the checklist of Tephritidae of Hungary (Diptera: Acalyptrata. Folia Entomologica Hungarica 61: 207-214.

- Merz B (2002) Tephritidae. In: Mason F, Cerretti P, Tagliapietra A, Speight M, Zapparoli M (Eds) Invertebrati di una foresta della Pianura Padana, Bosco della Fontana. Conservazione habitat Invertebrati 1. Arcari Editore. 122 pp.

- Merz B, Kofler A (2008) Fruchtfliegen aus Osttirol und Krnten (sterreich) (Diptera: Tephritidae). Linzer Biologische Beitrge 40 (2): 1211-1224.

- Merz B, Korneyev V (2011) Fauna Europaea: Tephritidae. Fauna Europaea version 2.4. https://fauna-eu.org

- $\quad$ Mihalyi F (1960) Frlegyek. Trypetidae. Magyarorszg llatvilga 15 (3): 1-76. 
- Mohamadzade Namin S, Nozari J, Rasoulian G (2010) The fruit flies (Diptera, Tephritidae) in Tehran Province, with new records for Iranian fauna. Vestnik Zoologii 44 (1): 23-34.

- Mohamadzade Namin S, Nozari J (2011) The fruit flies (Diptera: Tephritidae) in Kurdistan province, with new records for Iranian fauna. Ukrainska Entomofaunistyka 2 (4): 47-53.

- Norrbom AL, Carroll LE, Thompson FC, White IM, A F (1999) Systematic database of names (pp.). In: Thompson FC (Ed.) Fruit fly Expert Identification System and Systematic Information Database. Myia, 9. Backhuys Publishers, Leiden, 299 pp.

- $\quad$ Nugnes F, Russo E, Viggiani G, Bernardo U (2018) First record of an invasive fruit fly belonging to Bactrocera dorsalis complex (Diptera: Tephritidae) in Europe. Insects 9 (4). https://doi.org/10.3390/insects9040182

- $\quad$ Pignatti S (1982) Flora d'Italia. Edagricole, Bologna.

- Richter VA (1988) Family Tephritidae (Trypetidae). In: Bei-Bienko GY (Ed.) Keys to the insects of the European part of the USSR. Diptera and Siphonaptera. Part 2. Volume 5. Smithsonian Institution Libraries and National Science Foundation, Washington, 212-276 pp.

- $\quad$ Rivosecchi L (2008) Aggiunte e correzioni alle checklist di alcune famiglie di Ditteri della fauna italiana (Diptera). Bollettino della Società Entomologica Italiana 140 (2): 95-103.

- Rondani C (1856) Dipterologiae Italicae prodromus. Vol. I: Genera Italica ordinis dipterorum ordinatim disposita et distincta et in familiae et stirpes aggregata. A. Stocchi, Parmae [= Parma],228 + [2] pp.

- Rondani C (1870) Dipterologiae Italicae prodromus. Genera Italica ordinis dipterorum ordinatim disposita et distincta et in familias et stirpes aggregata. 7 (pars 4) (sect. 1). A. Stocchi, Parmae [= Parma],59 pp.

- $\quad$ Roser KL (1840) Erster Nachtrag zu dem im Jahre 1834 bekannt gemachten Verzeichnisse in Wurttemberg vorkommender zweiflugliger Insekten.

Correspondenzblatt des Koeniglich Wuerttembergischen Landwirthschaftlichen Vereins, Stuttgart 37 (1): 49-64.

- Séguy E (1934) Trypetidae. Faune de France 28, Dipteres (Brachyceres: Acalypterae et Scatophagidae). 28. Lechevalier et Fils, Paris, 832 pp.

- Seljak G (2013) The burr-seed fly, Euaresta aequalis (Loew) (Diptera: Tephritidae), newly recorded in Europe, with new observations on its biology. Studia Dipterologica 20 (1): 31-38.

- Shcherbakov MV (2001) Three new species of the genus Tephritis Latreille (Diptera, Tephritidae) from Southern Siberia. International Journal of Dipterological Researches 12 (2): 79-89.

- White IM (1988) Tephritid flies (Diptera: Tephritidae). Handbooks for Identification of British Insects. 10(5a). Royal Entomological Society of London, London, 134 pp.

- White IM, Korneyev VA (1989) A revision of the western Palaearctic species of Urophora Robineau-Desvoidy (Diptera: Tephritidae. Systematic Entomology 14: 327-374. https://doi.org/10.1111/j.1365-3113.1989.tb00289.x

- Zarghani E, Khaghaninia S, Mohamadzade Namin S, Karimpour Y, Korneyev VA (2016) First records of the fruit flies (Diptera, Tephritidae) in the fauna of Iran. Vestnik Zoologii 50 (2): 123-134. https://doi.org/10.1515/vzoo-2016-0015 